\title{
Fitossociologia e expectativa de renda com produtos florestais madeireiros e não madeireiros em assentamento no Pará
}

\author{
Josiel Carneiro PINHEIRO ${ }^{1 *}$, João Ricardo Vasconcellos GAMA ${ }^{1}$, Francisco de Assis OLIVEIRA ${ }^{2}$, \\ Renato Bezerra da SILVA RIBEIRO ${ }^{1}$, Girlene da Silva CRUZ $^{3}$
}

\author{
${ }^{1}$ Universidade Federal do Oeste do Pará, Santarém, PA, Brasil. \\ ${ }^{2}$ Universidade Federal Rural da Amazônia, Belém, PA, Brasil. \\ ${ }^{3}$ Universidade do Estado de Santa Catarina, Lages, SC, Brasil. \\ *E-mail: josielpinheiro@gmail.com
}

Recebido em abril/2018; Aceito em setembro/2018.

\begin{abstract}
RESUMO:O objetivo deste estudo foi analisar a estrutura e a expectativa de renda gerada pelas espécies arbóreas, considerando produtos florestais madeireiros e não madeireiros nos municípios de Santarém e Placas, Pará (PA). A área de estudo foi na comunidade São Mateus, Projeto de Assentamento do INCRA Moju I e II, município de Placas, PA. Empregou-se inventário florestal com amostragem sistemática de 11 unidades amostrais de área fixa de $50 \mathrm{~m}$ x $200 \mathrm{~m}$, totalizando 11 ha. Todos os indivíduos com DAP $\geq 10 \mathrm{~cm}$ foram mensurados, considerando diferentes classes de tamanho (CT) e subamostragens. Foram mensurados 903 indivíduos com DAP $\geq 10 \mathrm{~cm}$ (501,6 árv.ha $\left.{ }^{-1}\right)$, distribuídas em 48 famílias e 143 espécies botânicas. As famílias botânicas que apresentaram maior riqueza de espécies foram: Fabaceae (36), Sapotaceae (13), Lecythidaceae (10), Malvaceae (7), Moraceae (7), Annonaceae (5), Burseraceae (5) e Lauraceae (5). As famílias representam 61,5\% do número de espécies, confirmando-se que poucas famílias botânicas representam um grande número de espécies em Floresta Ombrófila Densa. O Coeficiente de mistura de Jentsch (QM) apresentou em média 6,31 indivíduos por espécie; a área basal foi de $28,77 \mathrm{~m}^{2} \cdot \mathrm{ha}^{-1}$; o índice de Shannon Weaver ( $\mathrm{H}^{\prime}$ ) 4,28; a diversidade Máxima (H'máx) 4,96 e a Equabilidade de Pielou (J) 0,86. A floresta não manejada apresentou estoque de espécies com potencial madeireiro e não madeireiro. As atividades de extração de madeira e PFNMs podem gerar lucros por hectare superiores ao salário mínimo e ajudar no complemento de renda das famílias assentadas.
\end{abstract}

Palavras-chave: Fabaceae, manejo florestal, recursos florestais.

\section{Phytosocyology and expectation of income with wood and non-gradual forest products in nesting in Pará}

\begin{abstract}
The objective of this study was to analyze the structure and income expectation generated by tree species, considering timber and non-timber forest products in the municipalities of Santarém and Placas, Pará (PA). The study area was in the São Mateus community, the Settlement Project of INCRA Moju I and II, municipality of Placas, PA. The study area was in the São Mateus community, the Settlement Project of INCRA Moju I and II, municipality of Placas, PA. A forest inventory was used with systematic sampling of 11 sample units of fixed area of $50 \mathrm{~m} \times 200 \mathrm{~m}$, totaling $11 \mathrm{ha}$. All individuals with $\mathrm{DBH} \geq 10 \mathrm{~cm}$ were measured, considering different size classes (CT) and sub-samples. A total of 903 individuals with $\mathrm{DBH} \geq 10 \mathrm{~cm}(501.6$ tree-ha-1) were distributed in 48 families and 143 botanical species. The botanical families with the highest species richness were Fabaceae (36), Sapotaceae (13), Lecythidaceae (10), Malvaceae (7), Moraceae (7), Annonaceae (5), Burseraceae (5) and Lauraceae. The families represent $61.5 \%$ of the number of species, confirming that few botanical families represent a large number of species in DenseOmbrophylousForest. The Jentsch mixing coefficient (QM) presented, on average, 6.31 individuals per species; the basal area was $28.77 \mathrm{~m}^{2} \cdot \mathrm{ha}^{-1}$; the Shannon Weaver index (H ') 4.28; the Maximum diversity (H'max) 4.96 and the Pielou Equability (J) 0.86 . The unmanaged forest presented stock of species with potential lumber and not timber. Logging activities and NTFPs can generate profits per hectare above the minimum wage and help supplement the incomes of settled families.
\end{abstract}

Keywords: Fabaceae, forest management, forest Resources.

\section{INTRODUÇÃO}

Os recursos naturais, durante muito tempo, foram considerados infindáveis e não havia preocupação quanto à sustentabilidade dos sistemas de exploração. Atualmente, a humanidade procura meios para atender à necessidade eminente por estes recursos e conservá-los para as futuras gerações. No Brasil, mais especificamente na Amazônia, maior floresta tropical do mundo, a exploração dos recursos florestais é a base da economia de vários municípios, sendo a única fonte de renda de muitas famílias. No entanto, esta atividade está longe de alcançar a sustentabilidade econômica e ecológica, e apesar de algumas empresas utilizarem o Manejo Florestal, a exploração predatória e ilegal predominam na região (GAMA et al., 2011).

A floresta a tempos atrás era vista como algo sem valor ou que atrapalhava o desenvolvimento, que deveria ser removida 
para dar lugar às atividades conhecidas rentáveis, como a pecuária, culturas de cana, milho, feijão, arroz, café, e agora a soja. Quando os produtores rurais perceberem que o aproveitamento da floresta pode permitir atividades rentáveis, haverá maior esforço por parte destes para a conservação dos recursos.

Segundo Gama et al. (2011), as iniciativas governamentais de fomento à produção florestal em bases sustentáveis fazem do manejo dos ecossistemas florestais um instrumento na busca pelo equilíbrio entre demanda e oferta. Desse modo, ao respeitar a capacidade produtiva da floresta o manejador torna possível a exploração sucessiva de uma mesma unidade de exploração, respeitando os ciclos de corte e garantindo a conservação de sua fonte de renda. As florestas são ambientes complexos que exigem estudos aprofundados para o seu entendimento. A análise da estrutura das florestas nativas deve iniciar com uma abordagem da composição florística e das estruturas horizontal, vertical, contemplando as estimativas de distribuição de diâmetros, área basal e volume (SOUZA, 2003).

A floresta é uma fonte de variados recursos, considerando os princípios do manejo florestal, estando a eficiência de sua utilização diretamente relacionada ao valor que lhe é atribuída. Logo, o conhecimento da potencialidade dos ecossistemas florestais é de fundamental importância, porque torna possível a seleção de áreas com melhores possibilidades de uso, alocação de infraestrutura e definição das atividades a serem desenvolvidas, adequadas às características do meio físico, sem riscos de causar alterações ambientais graves. É senso comum que as florestas somente transformam-se em riqueza gerada pelas atividades agrícolas quando exploradas racionalmente (OLIVEIRA et al., 1993). Todavia, é necessário que a utilização seja feita com conhecimento técnico comprovado pela pesquisa, a qual associe tecnologia com planejamento.

Neste cenário os benefícios oriundos da madeira comercial constituem apenas um subsistema de produção e na verdade outros recursos florestais precisam ser considerados para o aproveitamento integral nos ecossistemas, tais como o subsistema de produtos não madeireiros, como componente essencial da integralização do sistema de produção, numa conjuntura de uso sustentável dos recursos naturais (OLIVEIRA et al., 1993).

A valoração da floresta, considerando os produtos florestais comercializados em cada região, é uma ação de fácil execução e fundamental para se verificar as possibilidades de receita. Gera ainda informações úteis para análise da viabilidade econômica da utilização do recurso florestal, sendo uma medida importante na avaliação da economicidade das opções de manejo e fator fundamental a ser considerado por ocasião da comercialização de propriedades rurais e da fiscalização ambiental pelos órgãos competentes (OIMT, 1990).

Vale destacar que na determinação das ações futuras de manejo da floresta, em que pese a necessidade de um planejamento criterioso das intervenções, a fim de assegurar a sustentabilidade, a análise fitossociológica associada à valoração da floresta são ferramentas importantes e viáveis na obtenção de informações sobre o povoamento (BENTESGAMA et al., 2002).

Portanto, o conhecimento da composição florística, estrutura e produtividade da floresta são fundamentais para o uso sustentável tanto do ponto de vista ecológico quanto do econômico. Deste modo, o objetivo deste estudo foi analisar a estrutura da floresta e a expectativa de renda na Comunidade São Mateus, gerada pelas espécies arbóreas, considerando produtos florestais madeireiros e não madeireiros com demanda de comercialização na região dos municípios de Santarém e Placas, Pará.

\section{MATERIAL E MÉTODOS}

\section{1. Área de estudo}

Este estudo foi realizado na área de floresta da comunidade São Mateus, localizada no Projeto de Assentamento Moju I e II, na região Oeste do Estado do Pará. A comunidade tem uma superfície de cerca de 2.431 hectares, localizada na vicinal do km 145 da BR-163, município de Placas, PA, Latitude: 332'58.89” e Longitude: -54²3'57.11" (Figura 1). Fundada em 1985, residem 40 famílias, com cerca de 280 pessoas. Possui uma escola multiseriada que atende 36 crianças de $1^{\mathrm{a}}$ a $4^{\mathrm{a}}$ série do ensino fundamental. Além disso, possui uma Igreja evangélica e uma Associação de Pequenos Produtores Rurais (APROCOSMA), com 32 sócios. Não possui agrovila, posto de saúde e nem telefone público, apenas um barracão comunitário. A maioria das residências são construções de madeira e barro, com piso de cimento queimado e terra batida, respectivamente, e com cobertura de cavaco/madeira. Seus principais produtos agrícolas são farinha de mandioca e pimenta-do-reino (GAMA et al., 2011).
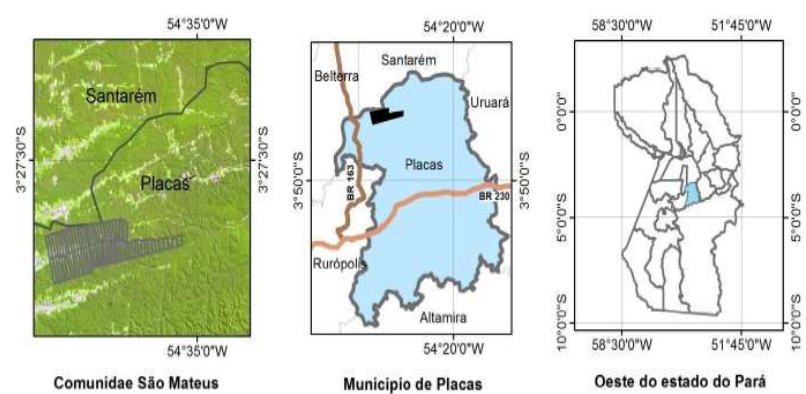

Figura 1. Comunidade São Mateus, PA Moju I e II, município de Placas, Oeste do Pará.

Figure 1. Community of São Mateus, PA Moju I and II, municipalityof Placas, West of Pará.

O clima da região do Baixo Tapajós é do tipo Afi, de acordo com a classificação climática de Köppen, caracterizado por climas tropicais úmidos, sem estação fria, com temperatura quente de baixa amplitude anual, apresentando mínima média anual de $16{ }^{\circ} \mathrm{C}$ e máxima média anual de $34{ }^{\circ} \mathrm{C}$, com umidade relativa média de $91 \%$. A precipitação é do tipo convectiva, em forma de pancadas de curta duração, com médias anuais oscilando em torno de $2.000 \mathrm{~mm}$. O trimestre mais seco ocorre de setembro a novembro e o trimestre mais chuvoso vai de fevereiro a abril (SUDAM, 1984; FUNDAC, 2005).

A vegetação é do tipo Floresta Ombrófila Densa de terra firme (IBGE, 2012). Neste tipo de vegetação ocorrem árvores de grande porte, trepadeiras lenhosas e epífitas em abundância, podendo ocorrer período seco de até 60 dias por ano (IBGE, 1992). A floresta já sofreu exploração seletiva, não-autorizada, com retirada de espécies madeireiras de valor comercial (Handroanthus sp. e Cedrella sp.) para o abastecimento de serrarias da região.

O solo presente na comunidade São Mateus é ácido, profundo e de atividade físico-química muito baixa constituído 
por material mineral, apresentando horizonte B latossólico imediatamente abaixo de qualquer tipo de horizonte A denominado de Latossolo Amarelo Distrófico (GAMA et al., 2011).

\subsection{Amostragem e coleta de dados}

O inventário florestal foi realizado por meio de amostragem sistemática de 11 unidades de amostra de área fixa de $50 \mathrm{~m}$ x $200 \mathrm{~m}$, sendo que cada unidade de amostra foi alocada em um lote, totalizando amostra de 11,0 ha. Foram incluídas no inventário florestal todas as espécies arbóreas com Diâmetro à Altura do Peito (1,30 $\mathrm{m}$ do solo) - DAP igual ou superior a $10 \mathrm{~cm}$. Os indivíduos foram mensurados considerando as seguintes classes de tamanho: (50 m x $200 \mathrm{~m}$, todos os indivíduos que apresentaram DAP $\geq 50 \mathrm{~cm}$ (classe de tamanho - CT3), em subparcelas de $50 \mathrm{~m} \times 40 \mathrm{~m}$, os indivíduos com $30 \mathrm{~cm} \leq \mathrm{DAP}<50 \mathrm{~cm}(\mathrm{CT} 2)$ e, em subparcelas de $50 \mathrm{~m}$ x $20 \mathrm{~m}$, os indivíduos com $10 \mathrm{~cm} \leq$ DAP $<30 \mathrm{~cm}$ (CT1)).

Foi registrado o nome regional de cada indivíduo, DAP, altura comercial (Hc), qualidade de fuste: $1=100 \%$ a $90 \%$ de aproveitamento, $2=90 \%$ a $70 \%$ de aproveitamento, $3=70 \%$ a $50 \%$ de aproveitamento e $4=$ menos que $50 \%$ de aproveitamento.

A identificação taxonômica foi realizada por meio de comparações no acervo de plantas da Universidade Federal do Oeste do Pará, com auxílio de especialistas. Para apresentação dos táxons, adotou-se o sistema de classificação proposto por Cronquist (1988), exceto para o grupo das Fabaceas, que foi tratado como uma única família.

Para a valoração dos produtos florestais, realizou-se entrevistas com pessoas da comunidade de São Mateus no município de Placas e nas feiras livres de Santarém para saber o uso comercial, produtividade e preço de mercado das espécies, as quais foram classificadas nos seguintes grupos: madeireiro, frutífera e fármaco. A escolha do município de Santarém para realização das entrevistas se deu pelo fato de toda a produção adquirida pela comunidade ser vendida nas feiras livres da cidade, por causa da via de acesso e a presença de transportes coletivos frequentes na região do Moju I e II. Ao contrário do município de Placas que apesar de ser mais próximo da comunidade, a via de acesso é precária, sem pavimentação e não há transporte coletivo, dificultando o escoamento dos produtos agrícolas para a venda.

\subsection{Análise e processamento dos dados}

A diversidade de espécies foi estimada por meio do índice de Shannon-Weaver. A equabilidade de Pielou que estima o nível de representatividade do índice de diversidade e, o coeficiente de mistura de Jentsch que representa o número médio de indivíduos por espécie foram estimados conforme recomendação de Brower; Zar (1984).

Os parâmetros fitossociológicos da estrutura horizontal (densidade, dominância, frequência e valor de importância) foram calculados de acordo com Curtis; Mcintosh (1951) e a posição sociológica, que se refere à participação das diferentes espécies em cada estrato da floresta, foram calculados conforme Finol (1971).

O método de estratificação utilizado foi o recomendado por Souza (1990) que se baseia no desvio padrão das alturas das espécies observadas no inventário florestal, a saber: Estrato inferior - compreende as árvores com altura total $(\mathrm{H})$ menor que a altura média $(\mathrm{Hm})$ menos uma unidade de desvio padrão $(1 \sigma)$ das alturas totais, ou seja, $\mathrm{H}<(\mathrm{Hm}-1 \sigma)$; Estrato médio - compreende as árvores com $(\mathrm{Hm}-1 \sigma) \leq \mathrm{H}<(\mathrm{Hm}+$ $1 \sigma)$; e Estrato superior - compreende as árvores com $\mathrm{H} \geq(\mathrm{Hm}$ $+1 \sigma)$.

Para analisar a distribuição diamétrica, as árvores com diâmetro igual ou maior que o nível de inclusão de DAP foram agrupadas em classes, com $10 \mathrm{~cm}$ de amplitude, visto que a floresta estudada se encontra em estágio de regeneração secundário avançado (SOUZA, 2001). A estratificação da floresta também foi analisada por meio da distribuição de alturas, adotando-se amplitude de $4 \mathrm{~m}$ para cada classe.

$\mathrm{O}$ volume de fuste comercial com casca de árvores em pé da i-ésima espécie $\left(\mathrm{Vc}_{\mathrm{i}}\right)$ foi estimado utilizando o fator de forma igual a 0,7 (HEINSDIJK; BASTOS, 1963), por meio da seguinte equação 1 :

$$
V c=\frac{\pi}{40000} * D A P^{2} * H c * 0,7
$$

em que: $H c=$ altura comercial, em m; $0,7=$ fator de forma; $D A P=$ diâmetro medido a $1,30 \mathrm{~m}$ do solo; $\pi=3,14159265$.

\subsection{Valoração dos produtos florestais}

Por meio da valoração dos produtos que existem na floresta, estimou-se a expectativa de renda considerando a área reserva legal das propriedades que é de, aproximadamente, 80 ha, avaliando apenas o estoque de exploração (DAP $\geq 50 \mathrm{~cm}$ ) e valor de mercado das espécies encontradas na região tanto para madeira, quanto para os produtos não madeireiros.

Para estimativa de produção madeireira adotou-se intensidade de corte de $10 \mathrm{~m}^{3} \cdot \mathrm{ha}^{-1}$ prevendo o manejo da área de reserva legal por dez anos e, desta forma a área total manejada seria de 8 ha.

$\mathrm{Na}$ escolha das espécies e árvores para a estimativa de volume de madeira a ser valorado, utilizou-se os critérios de seleção de árvores para corte e manutenção recomendado pelos órgãos ambientais (federal e estadual), publicados nos documentos IN/MMA nº5 (BRASIL, 2006), a saber: espécies que estão sendo comercializadas na atualidade, diâmetro mínimo de corte igual ou maior que $50 \mathrm{~cm}$ e classe de qualidade de fuste tipo 1, ou seja, com $100 \%$ a $90 \%$ de aproveitamento. Dentre as árvores, que atenderam os critérios de seleção para corte, foram mantidas $10 \%$, respeitou-se a manutenção de no mínimo 3 árvores por espécie por 100 ha e a intensidade máxima de corte de $10 \mathrm{~m}^{3} \cdot \mathrm{ha}^{-1}$, conforme IN/MMA nº 05 (BRASIL, 2006).

A expressão matemática para calcular o valor de fuste das espécies comerciais foi de acordo com a equação 2, enquanto que o valor monetário dos produtos da floresta foi obtido pela equação 3 .

$$
V M_{E C}=\sum_{i=1}^{S} V F E_{i} . P M E_{i}
$$

em que: $V M_{E C}=$ valor monetário de fuste das espécies comerciais, em R $\$ . U_{P A}^{-1}$ (Unidade de Produção Anual); $V F E_{\mathrm{i}}=$ volume de fuste do estoque de exploração (DAP $\geq 50 \mathrm{~cm}$ ) da i-ésima espécie comercial selecionada para colheita, em m${ }^{3} \mathrm{UPA}^{-1} ; P M E_{\mathrm{i}}=$ preço da madeira em pé da i-ésima espécie comercial, em $\mathrm{R} \$ \mathrm{~m}^{-3} ; \mathrm{S}$ = número de espécies.

$$
V M_{P F}=V M_{E C}+V M_{P F N M}
$$

em que: $V M P F=$ valor monetário dos produtos da floresta, em $\mathrm{R} \$$ ha ${ }^{1}$; e $V M E C, V M P F N M=$ já definidos anteriormente. 
A tabulação e o processamento dos dados foram realizados por meio da planilha eletrônica do Excel ${ }^{\circledR} 2010$.

\section{RESULTADOS}

3.1. Composição florística e diversidade das espécies

No inventário florestal foram mensurados 903 indivíduos com DAP $\geq 10 \mathrm{~cm}\left(501,6\right.$ árv.ha $\left.{ }^{-1}\right)$, distribuídas em 48 famílias e 143 espécies botânicas. As famílias botânicas que apresentaram maior riqueza de espécies foram: Fabaceae (36), Sapotaceae (13), Lecythidaceae (10), Malvaceae (7), Moraceae (7), Annonaceae (5), Burseraceae (5) e Lauraceae (5).

A floresta apresentou diversidade de $\mathrm{H}^{\prime}=4,28$, o índice de equabilidade de Pielou (J) foi de $86 \%$, ou seja, a diversidade máxima possível alcançada na amostra e coeficiente de mistura de Jentsch (QM) apresentou em média 6 indivíduos por espécie (Tabela 1).

Tabela 1.Índices estruturais e de diversidade para árvores com DAP $\geq 10 \mathrm{~cm}$ amostradas em 11 ha de floresta não manejada, Comunidade São Mateus, Placas, Pará.

Table 1. Structural and diversity indexes for treeswith $\mathrm{DBH} \geq 10 \mathrm{~cm}$ sampled in 11 ha of un managed forest, São Mateus Community, Plate, Pará.

\begin{tabular}{lc}
\hline \multicolumn{1}{c}{ Parâmetro } & $\begin{array}{c}\text { Floresta Não } \\
\text { Manejada }\end{array}$ \\
\hline Amostra (ha) & 11 \\
Número de famílias & 48 \\
Riqueza de espécies (S) & 143 \\
Densidade absoluta (ind.ha $\left.{ }^{-1}\right)$ & 501,64 \\
Área basal $\left(\mathrm{m}^{2}\right.$.ha $\left.^{-1}\right)$ & 28,77 \\
Índice de Shannon Weaver (H') & 4,28 \\
Diversidade Máxima (H' máx.) & 4,96 \\
Equabilidade de Pielou (J) & 0,86 \\
Coeficiente de Mistura de Jentsch (QM) & 6,31 \\
\hline
\end{tabular}

\subsection{Análise da Estrutura Arbórea}

Foram estimados 501,6 ind.ha ${ }^{-1}$ que totalizaram área basal de $28,8 \mathrm{~m}^{2} \cdot \mathrm{ha}^{-1}$ nos 11 ha inventariados. A estrutura diamétrica apresentou tendência a J-invertido, que é o padrão característico das florestas inequiâneas (Figura 2). O diâmetro máximo encontrado foi de 135,9 cm em Aspidosperma sp.

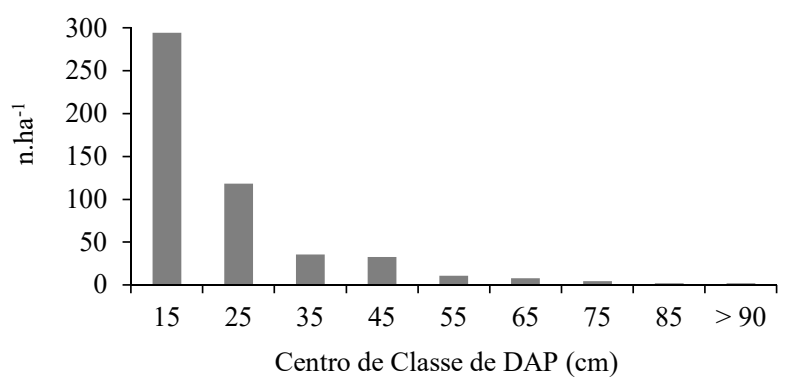

Figura 2. Distribuição do número de indivíduos por hectare e por classe diamétrica, comunidade São Mateus, município de Placas, PA. Figure 2. Distribution of the number of individuals per hectare and by diameter class, community of São Mateus, municipality of Placas, PA.

Foi possível observar que o estoque de exploração (DAP $\geq$ $50 \mathrm{~cm}$ ) foi de 27,1 ind. ha ${ }^{-1}$, em termos de proporcionalidade ocuparam 5,3 do total de árvores da floresta. E o estoque de crescimento $(10 \mathrm{~cm} \leq$ DAP $<50 \mathrm{~cm})$ de 480,9 ind. ha ${ }^{-1}$. Na distribuição das árvores por classes de altura verificou-se que
$70,6 \%$ dos indivíduos se concentraram nas classes $12 \mathrm{~m}$ e 16 $\mathrm{m}$ (Figura 3). A altura média encontrada foi $17,5 \mathrm{~m}$ e os resultados do valor fitossociológico foi de $4 \%$ dos indivíduos estavam concentrados no estrato inferior, $79 \%$ no estrato médio e $17 \%$ no estrato superior.

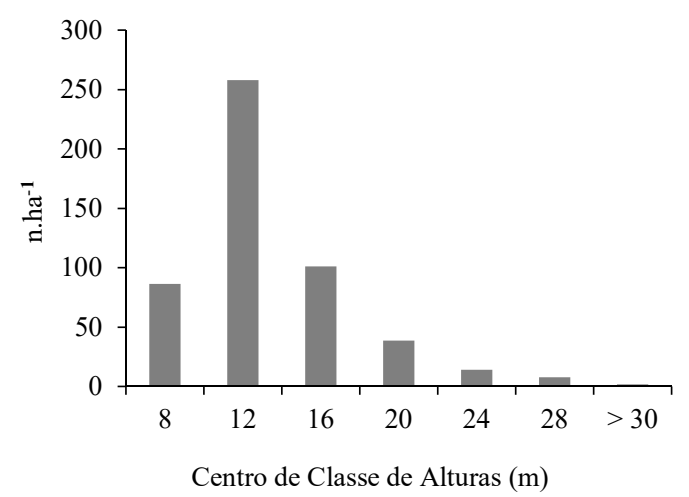

Figura 3. Distribuição do número de indivíduos por hectare (ind.ha ${ }^{-1}$ ) e por classe de altura, comunidade São Mateus, município de Placas, PA.

Figure 3. Distribution of the number of individuals per hectare (ind.ha ${ }^{-1}$ ) and by height class, São Mateus community, municipality of Placas, PA.

\subsection{Estrutura horizontal e vertical da floresta}

Dentre as 143 espécies identificadas, apenas 89 apresentaram densidade absoluta (DA) igual ou superior a 1 ind.ha ${ }^{-1}$. As 10 espécies mais abundantes $\left(\mathrm{DA}>12\right.$ ind.ha $\left.^{-1}\right)$ foram Eschweilera coriácea (DC.) S.A. Mori, Nectandra sp., Bixa arbórea Huber, Protium CF. heptaphyllum (Aubl.) Marchand, Licania kunthiana Hook. f., Pouteria guianensis Aubl, Tetragastris altíssima (Aubl.) Swart, Pouteria cladanta Sandwith, Duguetia sp. e Pouteria macrophylla (Lam.) Eyma, que juntas, representaram $37,6 \%$ da densidade absoluta (Tabela 2).

Considerando as espécies com maiores valores de DA e que também se mostraram bem distribuídas na área, destacaram-se Tetragastris altissima, Nectandra sp., Pouteria guianensis, Eschweilera coriacea e Bixa arborea. A frequência de ocorrência destas espécies variou de 72,7 a $100 \%$. As 10 espécies de maior dominância absoluta (DoA> 0,67 $\mathrm{m}^{2} \cdot \mathrm{ha}^{-1}$ ) foram Mezilaurus itauba Taubert ex Mez., Manilkara bidentata (A.DC.) A.Chev., Manilkara huberi (Ducke) Chevalier, Pouteria guianensis Aubl., Eschweilera coriacea (DC.) S.A. Mori, Nectandra sp., Pouteria oppositifolia (Ducke) Baehni, Licania kunthiana Hook.f., Protium cf. heptaphyllum (Aubl.) Marchande Chamae cristascleroxylon (Ducke) H.S. Irwin \& Barneby, que em conjunto, representaram 38,6\% de dominância total (DoT).

Para verificar a ocorrência das espécies nos diferentes estratos da floresta, realizou-se a análise da posição sociológica relativa (PSR) e do valor de importância ampliado (VIA). As 30 espécies mais importantes quanto a estes parâmetros, juntas, representam $63,55 \%$ do VIA total da amostra. Eschweilera coriácea foi a mais importante (4,57\%), seguida por Mezilaurus itauba (3,82\%) e Nectandra sp. $(3,81 \%)$.

3.3. Valor monetário dos produtos da floresta

Verificou-se que as espécies Mezilaurus itauba, Hymenaea parviflora, Nectandra sp., Manilkara huberi, Manilkara bidentata e Stryphnodendron polystachyum 
apresentaram índices que as qualificam para compor o grupo de espécies para produção de madeira. As demais não apresentaram estoque de exploração ou qualidade de fuste tipo 1 (Tabela 3).

Para estimativa do valor monetário dos produtos da floresta (madeireiro e não madeireiros) e atender às exigências legais dos órgãos ambientais (BRASIL, 2006) para a colheita de madeira considerou-se uma Unidade de Manejo Florestal
(UMF) de 80 ha com Unidades de Produção Anual (UPA) de 8 ha, produção de madeira em ciclos de corte (intervalo entre colheitas) de 10 anos e produtividade máxima de $10 \mathrm{~m}^{3}$ de madeira por hectare (plano de manejo de baixa intensidade). Sendo assim, o comunitário poderia obter retorno financeiro com a madeira por um período de 10 anos e de não madeireiros de forma contínua, dependendo do tipo de produto utilizado.

Tabela 2. Parâmetros fitossociológicos em ordem decrescente de valor de importância ampliado em percentagem (VIA) para as 30 espécies mais importantes e percentual de ocorrência de árvores por qualidade de fuste no estoque de colheita (QFEC), comunidade São Mateus, município de Placas, PA.

Table 2. Phytosociological parameters in order of decreasing importance of importance value in percentage (VIA) for the 30 most important species and percentage of tree occurrence by stem quality in the harvesting stock (QFEC), community of São Mateus, municipality of Placas, PA.

\begin{tabular}{|c|c|c|c|c|c|c|c|c|c|c|}
\hline \multirow{2}{*}{ \# } & \multirow{2}{*}{ Espécie } & \multirow{2}{*}{ FA } & \multirow{2}{*}{ DA } & \multirow{2}{*}{ DoA } & \multirow{2}{*}{ VI } & \multirow{2}{*}{ VIA } & \multicolumn{4}{|c|}{$\mathrm{QF}_{\mathrm{EC}}(\%)$} \\
\hline & & & & & & & 1 & 2 & 3 & 4 \\
\hline 1 & Eschweilera coriacea (D.C.) S. A. Mori & 72,73 & 30,18 & 0,93 & 3,69 & 4,57 & 0,0 & 0,0 & 0,0 & 0,0 \\
\hline 2 & Mezilaurus itauba(Meisn.) Taub. ex Mez. & 90,91 & 12,00 & 2,53 & 4,49 & 3,82 & 47,4 & 29,8 & 14,0 & 8,8 \\
\hline 3 & Nectandra sp. & 90,91 & 23,00 & 0,83 & 3,25 & 3,81 & 33,3 & 66,7 & 0,0 & 0,0 \\
\hline 4 & Manilkara bidentata (A.DC.) A.Chev. & 100,00 & 12,45 & 1,67 & 3,60 & 3,32 & 70,4 & 25,9 & 3,7 & 0,0 \\
\hline 5 & Pouteria guianensis Aubl. & 90,91 & 17,36 & 1,14 & 3,23 & 3,26 & 0,0 & 0,0 & 0,0 & 0,0 \\
\hline 6 & Protium cf. heptaphyllum Aubl. & 72,73 & 18,64 & 0,68 & 2,64 & 3,10 & 0,0 & 0,0 & 0,0 & 0,0 \\
\hline 7 & Tetragastris altissima (Aubl.) Swart & 100,00 & 17,27 & 0,42 & 2,47 & 2,89 & 0,0 & 0,0 & 0,0 & 0,0 \\
\hline 8 & Licania kunthiana Hook. f. & 63,64 & 18,00 & 0,74 & 2,59 & 2,85 & 0,0 & 0,0 & 0,0 & 0,0 \\
\hline 9 & Bixa arbórea Huber & 72,73 & 21,36 & 0,54 & 2,65 & 2,63 & 0,0 & 0,0 & 0,0 & 0,0 \\
\hline 10 & Pouteria cladanta Sandwith & 72,73 & 16,18 & 0,61 & 2,38 & 2,55 & 0,0 & 0,0 & 0,0 & 0,0 \\
\hline 11 & Chamaecrista scleroxylon (Ducke) H.S. Irwin \& Barneby & 72,73 & 11,55 & 0,67 & 2,15 & 2,31 & 0,0 & 14,3 & 71,4 & 14,3 \\
\hline 12 & Manilkara huberi (Ducke) Chev. & 90,91 & 8,55 & 1,15 & 2,66 & 2,29 & 82,8 & 13,8 & 3,4 & 0,0 \\
\hline 13 & Duguetia sp. & 54,55 & 13,64 & 0,40 & 1,82 & 2,19 & 0,0 & 0,0 & 0,0 & 0,0 \\
\hline 14 & Pouteria macrophylla (Lam.) Eyma & 45,45 & 12,82 & 0,34 & 1,63 & 1,99 & 0,0 & 0,0 & 0,0 & 0,0 \\
\hline 15 & Dialium guianense (Aubl.) Sandwith & 81,82 & 7,64 & 0,56 & 1,84 & 1,79 & 0,0 & 0,0 & 0,0 & 0,0 \\
\hline 16 & Pouteria oppositifolia (Ducke) Baehni & 90,91 & 6,09 & 0,76 & 2,04 & 1,76 & 0,0 & 0,0 & 0,0 & 0,0 \\
\hline 17 & Tachigali vulgarisL.F. & 54,55 & 8,73 & 0,54 & 1,66 & 1,74 & 0,0 & 0,0 & 0,0 & 0,0 \\
\hline 18 & Tachigali sp. & 72,73 & 7,27 & 0,49 & 1,66 & 1,66 & 0,0 & 0,0 & 0,0 & 0,0 \\
\hline 19 & Eschweilera grandiflora (Aubl.) Sandwith & 63,64 & 8,55 & 0,37 & 1,53 & 1,64 & 0,0 & 0,0 & 0,0 & 0,0 \\
\hline 20 & Myrciaria floribunda (Cambess.) D. Legrand. & 36,36 & 10,00 & 0,26 & 1,27 & 1,56 & 0,0 & 0,0 & 0,0 & 0,0 \\
\hline 21 & Stryphnodendron polystachyum (Miq.) Kleinh. & 54,55 & 8,91 & 0,37 & 1,47 & 1,55 & 100,0 & 0,0 & 0,0 & 0,0 \\
\hline 22 & Zygia racemosa (Ducke) Barneby \& J.W. Grimes & 45,45 & 8,64 & 0,33 & 1,34 & 1,53 & 0,0 & 0,0 & 0,0 & 0,0 \\
\hline 23 & Naucleopsis sp. & 45,45 & 7,36 & 0,31 & 1,23 & 1,37 & 0,0 & 0,0 & 0,0 & 0,0 \\
\hline 24 & Lecythis sp. & 54,55 & 7,73 & 0,41 & 1,44 & 1,29 & 0,0 & 0,0 & 0,0 & 0,0 \\
\hline 25 & Ocotea guianensis Aubl. & 36,36 & 7,27 & 0,19 & 1,01 & 1,15 & 0,0 & 0,0 & 0,0 & 0,0 \\
\hline 26 & Couroupita guianensis Aubl. & 27,27 & 6,91 & 0,20 & 0,92 & 1,11 & 0,0 & 0,0 & 0,0 & 0,0 \\
\hline 27 & Alexa grandiflora Ducke & 54,55 & 3,09 & 0,42 & 1,15 & 1,03 & 0,0 & 0,0 & 0,0 & 0,0 \\
\hline 28 & Pseudopiptadenia suaveolens (Miq.) J.W. Grimes & 54,55 & 2,00 & 0,53 & 1,21 & 1,00 & 0,0 & 0,0 & 0,0 & 0,0 \\
\hline 29 & Rinorea neglecta Sandwith & 36,36 & 6,36 & 0,10 & 0,84 & 0,97 & 0,0 & 0,0 & 0,0 & 0,0 \\
\hline 30 & Hymenaea parviflora Huber & 54,55 & 2,00 & 0,36 & 1,00 & 0,84 & 42,9 & 42,9 & 14,3 & 0,0 \\
\hline & $\begin{array}{ll}\text { Subtotal } \\
\end{array}$ & & 341,55 & 18,88 & 60,90 & 63,55 & & & & \\
\hline & Outras espécies & & 160,09 & 9,89 & 39,10 & 36,45 & & & & \\
\hline & Total & & 501,64 & 28,77 & 100,0 & 100,0 & & & & \\
\hline
\end{tabular}

em que: $\mathrm{FA}=$ frequência absoluta; $\mathrm{DA}=$ densidade absoluta; DoA = dominância absoluta; VI = valor de importância, em porcentagem; VIA = valor de importância ampliado em percentagem.

Os preços por metro cúbico de fuste (árvore em pé) variaram de $\mathrm{R} \$ 31,30 \mathrm{R} \$ . \mathrm{m}^{-3}$ para Manilkara bidentata a $137,90 \mathrm{R} \$ \mathrm{~m}^{-3}$ para Mezilaurus itauba, com preço médio de $67,57 \mathrm{R} \$ \mathrm{~m}^{-3}$. Após seguir as restrições legais previstas na IN MMA $n^{\circ} 05$ foram selecionadas para colheita 11 árvores de três espécies comerciais com $60 \mathrm{~cm} \leq$ DAP $<80 \mathrm{~cm}$ que totalizaram 77,3 $\mathrm{m}^{3}$ e uma receita anual de $\mathrm{R} \$ 5.231,61$, para cada ciclo de corte com UPAs de 8 ha, se o comunitário vender a árvore em pé. Isso correspondeu a uma produtividade de 9,7 $\mathrm{m}^{3} \cdot \mathrm{ha}^{-1}$ e um valor de $\mathrm{R} \$ 653,95$ por hectare (Tabela 3 ).
Quanto à valoração dos produtos não madeireiros considerou-se quantificação de toda a UMF, ou seja, 80 ha de reserva legal e uma coleta anual desses produtos. Nas entrevistas foi possível verificar que $100 \%$ das espécies inventariadas apresentaram pelo menos um tipo de uso; $27,46 \%$ dois; e $8,4 \%$ três diferentes usos. As espécies que mereceram destaque, pelas alternativas de uso, foram Trattinnickia glaziovii utilizada para fins madeireiros, alimento para caça, medicinal e defumação, Hymenaea courbaril, Brosimum acutifolium e Caryocar villosum 
recomendadas para fins madeireiros, alimentação humana, alimento para caça e medicinal. Apenas Caryocar villosum apresentou PFNM com demanda de comercialização na zona urbana do município de Santarém, outras espécies que não apresentaram usos tão diversificados, mas que tem produtos que possuem demanda de comercialização pode ser observada na Tabela 4.0 valor monetário dos produtos florestais não madeireiros totalizou R\$13.994,50 para uma área de 80 ha, o que correspondeu a uma renda mensal de R\$ 1.166,21 associada com a comercialização de PFNM.

A mandioca (produção de farinha) juntamente com a pimenta é a principal fonte de renda para os comunitários de São Mateus. Dentre os assentados, 60\% afirmam retirar seu sustento, principalmente da renda da terra, compreendido como agricultura em regime de subsistência com a comercialização de excedentes. Conforme o padrão da agricultura familiar em toda a região: $19 \%$ das famílias retiram sua renda de atividades comerciais (informais) e serviços em geral, $13 \%$ afirmam ter salários associados a outras atividades, e $8 \%$ declaram ter nos benefícios e bolsas governamentais sua principal fonte de renda. O valor monetário dos produtos da floresta foi estimado em $\mathrm{R} \$ 19.226,11$, o que correspondeu a uma renda mensal de R\$1.602,17. Sendo 72,8 \% composta pela comercialização de PFNM e 27,2\% pela venda da árvore em pé.

Tabela 3. Valor monetário de fuste das espécies comerciais selecionadas para colheita em unidade de produção anual de oito hectares, comunidade São Mateus, município de Placas, PA.

Table 3. Monetary value of stem of commercial species selected for harvest in an annual production unit of eight hectares, São Mateus community, municipality of Placas, PA.

\begin{tabular}{|c|c|c|c|c|c|c|c|c|c|}
\hline \multirow{2}{*}{ Espécie } & & \multicolumn{5}{|c|}{ Centro de classe de DAP $(\mathrm{cm})$} & \multirow{2}{*}{ Total } & \multirow{2}{*}{$\begin{array}{c}\text { Preço } \\
\left(\mathrm{R} \$ \mathrm{~m}^{-3}\right)\end{array}$} & \multirow{2}{*}{$\begin{array}{l}V M_{E C} \\
(\mathrm{R} \$)\end{array}$} \\
\hline & & 55 & 65 & 75 & 85 & $>90$ & & & \\
\hline \multirow{4}{*}{ Mezilaurus itauba } & Rem. (n) & 9 & 14 & 6 & 5 & 2 & 36 & \multirow{4}{*}{137,90} & \multirow{4}{*}{$1.858,56$} \\
\hline & Matrizes (n) & 2 & & & & & 2 & & \\
\hline & Colheita (n) & & 2 & & & & 2 & & \\
\hline & $\mathrm{V}\left(\mathrm{m}^{3}\right)^{*}$ & & 13,5 & & & & 13,5 & & \\
\hline \multirow{4}{*}{ Manilkara huberi } & Rem. (n) & 6 & 6 & 2 & 1 & 1 & 16 & \multirow{4}{*}{68,60} & \multirow{4}{*}{$2.532,19$} \\
\hline & Matrizes (n) & 2 & & & & & 2 & & \\
\hline & Colheita (n) & & 2 & 3 & & & 5 & & \\
\hline & $\mathrm{V}\left(\mathrm{m}^{3}\right)$ & & 11,4 & 25,6 & & & 36,9 & & \\
\hline \multirow{4}{*}{ Manilkara bidentata } & Rem. (n) & 7 & 4 & 3 & 1 & & 15 & \multirow{4}{*}{31,30} & \multirow{4}{*}{840,86} \\
\hline & Matrizes (n) & 2 & & & & & 2 & & \\
\hline & Colheita (n) & & 2 & 2 & & & 4 & & \\
\hline & $\mathrm{V}\left(\mathrm{m}^{3}\right)$ & & 11,1 & 15,8 & & & 26,9 & & \\
\hline
\end{tabular}

$V M_{E C}=$ valor monetário de fuste das espécies comerciais, em R $\$ \mathrm{UPA}^{-1} ; \mathrm{Rem}=$ remanescentes; $\mathrm{V}=$ volume dos indivíduos em $\mathrm{m}^{3}$.

Tabela 4. Valor monetário dos produtos florestais não madeireiros em 2011 verificado em São Mateus que possuem mercado para comercialização, comunidade São Mateus, município de Placas, PA.

Table 4. Monetary value of non-timber forest products in 2011 verified in São Mateus that have market for commercialization, community of São Mateus, municipality of Placas, PA.

\begin{tabular}{|c|c|c|c|c|c|c|c|}
\hline Espécie & Nome Regional & Produto & PS & Unidade & $\begin{array}{c}\text { NA } \\
(80 \mathrm{ha})\end{array}$ & $\mathrm{PC}(\mathrm{R} \$)$ & $\begin{array}{c}\text { VMPFNM } \\
\left(\mathrm{R} \mathrm{UMF}^{-1}\right)\end{array}$ \\
\hline Dipteryx odorata Willd. & Cumarú & Semente & 15 & $\mathrm{~kg}$ & 58 & 4,50 & $3.915,00$ \\
\hline Brosimum parinarioides Ducke & Amapá-doce & Seiva & 7 & 1 & 50 & 7,00 & $2.450,00$ \\
\hline Carapa guianensis Aubl. & Andiroba & Óleo & 5 & 1 & 43 & 10,00 & $2.150,00$ \\
\hline Diplotropis purpúrea $(\mathrm{Rich})$ & Sucupira-amarela & Semente & 20 & $\mathrm{~kg}$ & 7 & 15,00 & $2.100,00$ \\
\hline Caryocar villosum (Aubl.) Pers. & Piquiá & Fruto & 35 & $\mathrm{~kg}$ & 21 & 2,50 & $1.837,50$ \\
\hline Bertholletia excelsa Bonpl. & Castanha-do-Pará & Semente & 18 & $\mathrm{~kg}$ & 14 & 2,50 & 630,00 \\
\hline Tetragastris altíssima (Aubl.) Swart & Breu-branco & Resina & 1 & $\mathrm{~kg}$ & 72 & 8,00 & 576,00 \\
\hline Lecythis pisonis Cambess & Castanha-sapucaia & Semente & 12 & $\mathrm{~kg}$ & 14 & 2,00 & 336,00 \\
\hline
\end{tabular}

em que: PS = produtividade média por espécie; NA = número de árvores na unidade de manejo florestal $(\mathrm{UMF}) ; \mathrm{PC}=$ preço de comercialização; $V M_{P F N M}=$ valor monetário dos produtos florestais não madeireiros (PFNM), considerando as árvores com DAP $\geq 30 \mathrm{~cm}$.

\section{DISCUSSÃO}

\subsection{Composição florística e diversidade das espécies}

As oito famílias mais abundantes deste estudo representam $61,5 \%$ do número de espécies, confirmando-se que poucas famílias botânicas representam um grande número de espécies na Floresta Ombrófila Densa (GONÇALVES; SANTOS, 2008; ALMEIDA et al., 2013).Em relação ao quociente de mistura de Jentsch, Finol (1975) afirma que em florestas naturais tropicais, o quociente de mistura seria de, aproximadamente, nove indivíduos por espécie, indicando alta heterogeneidade. $\mathrm{O}$ valor encontrado indica alta diversidade de espécies para a floresta. Almeida et al. (2013) encontraram sete indivíduos por espécie na comunidade Santo Antônio pertencente ao mesmo Projeto de Assentamento, enquanto Gonçalves; Santos (2008), na Floresta Nacional do Tapajós obtiveram 11 indivíduos por espécies.

Em relação ao índice de diversidade de Shannon EspíritoSanto et al. (2005) e Gonçalves; Santos (2008) encontraram valores semelhantes, sendo $H^{\prime}=4,14$ e $H^{\prime}=4,22$, respectivamente. De acordo com Knight (1975), o índice de diversidade de Shannon-Weaver para florestas tropicais amazônicas varia, normalmente, de 3,83 a 5,85. 


\subsection{Valor monetário dos produtos da floresta}

A análise dos parâmetros fitossociológicos permite inferir sobre distribuição, porte, densidade das espécies e a participação nos estratos da floresta, que são critérios ambientais que devem ser analisados na escolha das espécies madeireiras.

Conforme a Lei Federal no 12.651 (BRASIL, 2012), até $20 \%$ de uma propriedade rural pode ser destinada a uso alternativo do solo e $80 \%$ é denominada área de reserva legal, podendo ser utilizada sob regime de manejo florestal sustentável.

A espécie que apresentou maior $\mathrm{m}^{3}$ de fuste em pé foi a Mezilaurus itauba e de acordo com Gama et al. (2009) a espécie é uma das mais demandadas e valorizadas no município de Santarém, pois os estaleiros priorizarem a utilização desta espécie na construção de barcos.

Quanto a valoração das espécies que produzem produtos não madeireiros, a espécie que apresentou maior valor monetário foi Dipteryx odorata (cumarú) que tem suas sementes utilizadas nos tratamentos de pneumonia, asma, gripe, dor de cabeça e tosse, e em nível internacional, na indústria de perfumes, cosméticos, cigarros, entre outras (EMBRAPA, 2004). O comércio das sementes de cumaru apresenta uma cadeia produtiva consolidada, simples, impulsionada pela demanda internacional (Japão, França, Alemanha e China) e ligada à cadeia da Bertholletia excelsa (castanha-do-Brasil), ou seja, com o mesmo destino de comercialização, que é o principal produto florestal nãomadeireiro comercializado na região da Calha Norte do rio Amazonas (SILVA et al., 2010).

Vale ressaltar que a espécie Bertholletia excelsa apresenta elevada produção de frutos a cada quatro anos. Conforme Gama et al. (2009) a oscilação na produção de castanha-dobrasil ocorre, devido a sua autoecologia, ou seja, em um ano a produção é alta e no outro baixa. Considerando que existe flutuação na produção de frutos das espécies arbóreas na floresta (SHANLEY; MEDINA, 2005) foi contabilizado apenas $50 \%$ da produção, informada pelos comunitários, das espécies que o fruto ou semente são comercializados.

Também merecem destaque outras espécies que apresentaram alto valor monetário, como Brosimum parinarioides que tem a seiva (látex) utilizada para o tratamento de má digestão e infecção respiratória; Carapa guianensis, o óleo extraído da semente tem uso para inflamação, cicatrização, limpeza de pele e repelente; e Diplotropis purpurea, as sementes são utilizadas como chá para artrite, artrose e reumatismo.

No que diz respeito a renda média mensal das famílias do assentamento, essa está abaixo de um salário-mínimo vigente. Há famílias com renda mínima mensal de $\mathrm{R} \$ 180,00$ e o máximo registrado é apenas $\mathrm{R} \$ 930,00$, sendo esses os casos em que há ingressos da pecuária (GAMA et al., 2011).

A expectativa de renda gerada pelos produtos madeireiros e não madeireiros é, sem dúvida, um estímulo ao comunitário, como também ao investimento em pesquisa e tecnologia para um melhor aproveitamento de produtos florestais.

\section{CONCLUSÕES}

A floresta da Comunidade de São Mateus apresentou estoque de espécies com potencial madeireiro e não madeireiro, confirmando a hipótese do estudo.

A floresta é uma grande fornecedora de produtos não madeireiros (PFNMs), garantindo subprodutos como alternativa de renda. A ocorrência de espécies de uso múltiplo nos lotes da comunidade é um aspecto de importância a ser relacionada às suas funcionalidades à qualidade de vida e geração de renda aos comunitários.

O manejo das espécies não madeireiras que ocorrem na comunidade, gera benefícios para a população local, enquanto promove simultaneamente a conservação dos ecossistemas florestais.

O retorno econômico anual com o manejo dos PFNMs que se encontram nos lotes da comunidade, sobrepõe os benefícios anuais da produção de madeira ou da agricultura familiar do assentamento.

Apesar da diversidade dos PFNMs, são poucas as espécies comercializadas, além de possuírem venda limitada a poucos pontos nos mercados da região.

As atividades de extração dos produtos florestais geram ao produtor lucros por hectare superiores ao salário mínimo atual, no caso da madeira, utilizando unidades de produção anuais de 8 ha e 80 ha para coleta de PFNMs.

\section{RECOMENDAÇÕES}

A utilização das espécies madeireiras e não madeireiras no mercado atual é rentável à comunidade, desde que sejam mantidos seu planejamento e manejo adequado da floresta: sendo recomendável que espécies como Dipteryx odorata, Brosimum parinarioides, Carapa guianensis, Diplotropis purpurea,Caryocar villosum, Tetragastris altíssima e Lecythis pisonis, sejam removidas da lista de espécies de corte para fins madeireiros por possuírem mercado para comercialização de produtos florestais não madeireiros.

$\mathrm{O}$ estudo apresentado tem a utilidade de mostrar alternativa de geração de renda para a comunidade mantendo a floresta em pé, utilizando a produção de madeira com o manejo florestal sustentável e a coleta dos produtos florestais não madeireiros mais comercializados na região, que a floresta oferece.

Apesar dos comunitários afirmarem não terem custos para coletarem os PFNMs, considera-se que há despesas no que se refere desde a coleta até a sua venda como: o tempo gasto para coletar, material utilizado para extração, o deslocamento, diária, transporte para escoar para a cidade mais próxima e negociação. Esses devem ser levado em consideração para que o produto tenha um preço final no mercado.

\section{REFERÊNCIAS}

ALMEIDA, L. S.; GAMA, J. R. V.; OLIVEIRA, F. A.; FERREIRA, M. S. G.; GONÇALVES, D. C. M. Uso de Espécies da Flora na Comunidade Rural Santo Antônio, BR-163, Amazônia Brasileira. Floresta e Ambiente, Seropédica, v. 20, n. 4, p. 435-446, 2013. DOI: http://dx.doi.org/10.4322/floram.2013.044

BENTES-GAMA, M. M.; SCOLFORO, J. R. S.; GAMA, J. R. V.; OLIVEIRA, A. D. Estrutura e valoração de uma floresta de várzea alta na Amazônia. Cerne, Lavras, v. 8, n. 1, p. 88-102, 2002.

BRASIL. Ministério do Meio Ambiente. Instrução Normativa N. 5, de 11 de dezembro de 2006. Dispõe sobre procedimentos técnicos para elaboração, apresentação, execução e avaliação técnica de Planos de Manejo Florestal Sustentável - PMFS nas florestas primitivas e suas formas de sucessão na Amazônia Legal, e dá outras providências. Disponível em: 
$<$ http://www.diariodasleis.com.br/busca/exibelink.php?nu mlink=216088>. Acesso em 09 jun. 2016.

BRASIL. Lei $\mathbf{n}^{\mathbf{0}}$ 12.651, de 25 de Maio de 2012. Dispõe sobre a proteção da vegetação nativa; altera as Leis $n^{\circ} 6.938$, de 31 de dezembro de 1996, e 11.428 , de 22 de dezembro de 2006; revoga as Leis $n^{\circ} 4.771$, de 15 de setembro de 1965 , e 7.754 , de 14 de abril de 1989 , e a medida provisória $\mathrm{n}^{\circ}$ 2.166-67, de 24 de agosto de 2001; e dá outras providências. Disponível

$<$ http://www.planalto.gov.br/ccivil_03/_Ato20112014/2012/Lei/L12651.htm>. Acesso em: 01 de setembro de 2018.

BROWER, J. E.; ZAR, J. H. Field and laboratory methods for general ecology. 2. ed. Dubuque: Wm. C. Brown Publishers, 1984. $226 \mathrm{p}$.

CRONQUIST, A. The evolution and classification of flowering plants. 2. ed. New York: The New York Botanical Garden. 1988. 555 p.

CURTIS, J. T.; McINTOSH, R. P. An upland forest continuum in the prairie-forest border region of Wiscosin. Ecology, New York, v. 32, n. 3, p. 476-496, 1951. DOI: https://dx.doi.org/10.2307/1931725

EMBRAPA_EMPRESA BRASILEIRA DE PESQUISA AGROPECUÁRIA. Cumaru: Dipteryx odorata. Brasília: EMBRAPA, 2004. não paginado. (Espécies arbóreas da Amazônia, 7).

ESPÍRITO-SANTO, F. D. B.; SHIMABUKURO, Y. E.; ARAGÃO, L. E. O.; MACHADO, L. M. Análise da composição florística e fitossociológica da floresta nacional do Tapajós com o apoio geográfico de imagens de satélites. Acta Amazonica, Manaus, v. 35, n. 2, p. 155 173, 2005. DOI: http://dx.doi.org/10.1590/S004459672005000200006

FINOL, U. H. Nuevos parametros a considerarse en el analisis estructural de las selvas virgines tropicales. Revista Forestal Venezolana, Merida, v. 14, n. 21, p. 29-42, 1971.

FINOL, U. H. La silvicultura em La Orinoquia venezolana. Revista Forestal Venezolana, Merida, v.1 8, n. 25, p. 37 114, 1975.

FUNDAC_FUNDO DE DESENVOLVIMENTO E AÇÃO COMUNITÁRIA. Plano de Desenvolvimento do Projeto de Assentamento Moju I e II. Santarém: FUNDAC, 2005. $153 \mathrm{p}$.

GAMA, J. R. V.; MORAES, A.; CARVALHO, A. N.; RIBEIRO, A. S. S.; SANTOS, C. A. A.; MONTEIRO, R.; GOCH, Y. G. F. Estudo de impacto ambiental e proposta de desenvolvimento sustentável para o assentamento Moju I e II, município de Santarém-Pa. Santarém: UFOPA, 2011. $235 \mathrm{p}$.

GAMA, J. R. V.; SOUSA, S. M.; FURTADO, D. S. Diagnóstico da cadeia produtiva da castanha-do-brasil na microrregião do baixo amazonas. Santarém: ITESAM/TDP Florestal, 2009. 43 p.
GONÇALVES, F. G.; SANTOS, J. R. Composição florística e estrutura de uma unidade de manejo florestal sustentável na Floresta Nacional do Tapajós, Pará. Acta Amazônica, Manaus, v. 38, n. 2, p. 229-244, 2008. DOI: http://dx.doi.org/10.1590/S0044-59672008000200006

HEINSDIJK, D.; BASTOS, A. M. Inventários florestais na Amazônia. Boletim do Setor de Inventário Florestal, v. 6, p. 1-10, 1963.

IBGE_INSTITUTO BRASILEIRO DE GEOGRAFIA E ESTATÍSTICA. Manual técnico de vegetação brasileira. Rio de Janeiro: IBGE, 1992. 92p. (Manuais Técnicos de Geociências, 1).

IBGE INSTITUTO BRASILEIRO DE GEOGRAFIA E ESTATÍSTICA. Manual técnico da vegetação brasileira. 2. ed. rev. e aum. Rio de Janeiro: IBGE, 2012. $271 \mathrm{p}$.

KNIGHT, D. H. A phytosociological analysis of species-rich tropical forest on Barro Colorado Island, Panama. Ecological Monographs, Lawrence, v. 45, n. 3, p. 259284, 1975. DOI: https://dx.doi.org/10.2307/1942424

OIMT_ORGANIZAÇÃO INTERNACIONAL DE MĀDEIRA TROPICAL. Diretrizes da OIMT para o manejo sustentado de florestas tropicais naturais. Malásia: Forest Research Institute of Malasya (FRIM), 1990. 180p. (Série Técnica, 5).

OLIVEIRA, F. A.; MARQUES, L. C. T.; FERREIRA, C. A. $P$. Produtos não madeireiros da Floresta Nacional do Tapajós, Santarém, Pará, Brasil. Belém: FAO/IBAMA, 1993. $24 \mathrm{p}$.

SHANLEY, P.; MEDINA, G. Frutíferas e plantas úteis na vida Amazônica. Belém: CIFOR: IMAZON, 2005. 300 p.

SILVA, T. M.; JARDIM, F. C. S.; SILVA, M. S.; SHANLEY, P. O mercado de amêndoas de Dipteryx odorata (Cumarú) no estado do Pará. Floresta, v. 40, n. 3, p. 603-614, 2010. DOI: http://dx.doi.org/10.5380/rf.v40i3.18922

SOUZA, A. L. Estrutura, dinâmica e manejo de florestas tropicais. Viçosa: Universidade Federal de Viçosa, 2003. 122 p. (Notas de aula).

SOUZA, A. L. Análise multivariada para manejo de florestas naturais: alternativas de produção sustentada de madeiras para serraria. Floresta, Curitiba, v. 20, n. 1-2, p. 101-102, 1990.

SOUZA, A. L. Manejo Florestal: análise estrutural de florestas inequiâneas. In: CURSO DE INVENTÁRIO FLORESTAL APLICADO AO MANEJO DE FLORESTAS NATIVAS, 1., 2001, Viçosa. Anais... Viçosa: IEF/UFV, 2001. p. 114-135.

SUDAM_PROJETO DE HIDROLOGIA E CLIMATOLOGIA DA AMAZÔNIA. Atlas Climatológico da Amazônia Brasileira. Belém: SUDAM, 1984. 125p. 